\title{
EVALUATION OF THE EFFECT OF STRONTIUM CITRATE ON BONE CONSOLIDATION DURING MANDIBULAR DISTRACTION OSTEOGENESIS IN RABBITS (EXPERIMENTAL STUDY)
}

\author{
Shehab Y. Alansi ${ }^{1}{ }_{B D s}$, Mervet M. Khalil ${ }^{2 P h D}$, Marwa G. Noureldin ${ }^{3 P h D}$
}

Hagar S. Abdel Fattah ${ }^{4} P h D$.

\begin{abstract}
INTRODUCTION: Distraction osteogenesis is defined as a procedure that can lengthen bone gradually. Complications may occur during this time, including refractors, local infections and loosening of the distractor device or pin. The most important disadvantage applies to the extended period of consolidation stage. Several methods were tested to improve new bone development, including the use of different adjuvant treatments; in order to minimize the time needed to complete the mandibular distraction osteogenesis MDO phases. Strontium is a medication used for treatment of osteoporosis and in osteoporotic patients; it has been shown that bone formation is promoted, while bone resorption is reduced.

AIM OF THIS STUDY: This study aimed to assess the effects of strontium citrate during MDO in a rabbit model.

MATERIALS AND METHODS: Fourteen rabbits weighing 3.0-3.5kg were separated into 2 groups: group I; (control group), group II; (received strontium citrate). Surgical procedures were performed to place the distractor system in both groups. The distractor was activated after 5 days of latency stage. After 4 weeks, all rabbits were euthanized and the distracted mandibles were surgically removed and prepared for light microscopic examination and histomorphometry.

RESULTS: Comparing to the control group, histological results revealed thicker bone trabeculae in the study group with narrower bone marrow. The histomorphometric analysis also showed significantly higher percentage of new bone per field in the study group.

CONCLUSIONS: Strontium citrate supplementation could enhance bone consolidation throughout the distraction osteogenesis.

KEY WORDS: Strontium, Bone healing, Distraction osteogenesis, Mandible, Rabbits.

RUNNING TITLE: Effect of strontium citrate on DO in rabbits.
\end{abstract}

1 Bachelor of Dental Surgery, Faculty of Dentistry, Ibb University, Ibb, Yemen.

2 Professor of Oral and Maxillofacial Surgery, Alexandria University, Alexandria, Egypt.

3 Lecturer of Oral and Maxillofacial Surgery, Faculty of Dentistry, Alexandria University, Alexandria, Egypt.

4 Lecturer of Oral biology, Faculty of Dentistry, Alexandria University, Alexandria, Egypt.

* Corresponding author

E-mail: shehabalansy@gmail.com

\section{INTRODUCTION}

Ilizarov identified distraction osteogenesis in 1954 (1) and it became a standard technique in orthopedic surgery. Distraction osteogenesis (DO) has been commonly applied in craniomaxillofacial and orthopedic surgical procedures to treat bone defects and deformities (2). Craniofacial microsomia cases treated with mandible distraction were first presented by McCarthy et al in craniofacial surgery (3). Since then, several series of craniofacial hypoplasia, including mandible, maxilla, and others, have been treated with bone distraction (4-7).

Distraction osteogenesis is defined as procedure that can lengthen bone gradually (8). In the postsurgical period, mandibular distraction osteogenesis is applied in three stages: latency stage, which is the period from bone division to the onset of traction and represents the time required for reparative formation between the osteotomized bone segments; distraction stage, which is that time when a traction force is applied to bone segments and a new bone is formed within inter-segmentary gap. Finally, consolidation stage is the period that allows mineralization and cortication of the newly formed bone tissue prior to device removal. During the latency phase the osteotomy gap is at first occupied with callus or fibrous tissue, which later is stretched by gradual forces during the distraction stage, finally and during the consolidation stage the callus tissue then is remodeled by mature bone (9).

While MDO has shown advances in upper airway obstruction associated with swallowing difficulties (10) and micrognathia (11), there are restrictions. The most important disadvantage applies to the extended period of consolidation stage. Many complications may occur during this time, including refractures, local infections and loosening of the distractor device or pin. Several methods have been tested for their potential to increase new bone development, including the use of different adjuvant treatments; in order to minimize the time needed to complete the MDO phases (12). 
While some of these were effective in initial trials, in the clinical setting, there is actually no adjuvant treatment, since most are either considered to be investigational [e.g. gene therapy] (13) or impractical [since more procedure are required (14), regular hyperbaric oxygen application with possible risk of oxygen poisoning and lung damage (15)].

Other adjuvant medication was used such as bisphosphonates (the disadvantage of this medication that it is associated with an increased risk of atypical hip fractures), estrogen (risk of blood clots) and denosumab (an inhibitor of receptor activator of nuclear factor-kB ligand), which can be given orally or parentally (16).

Strontium is a medication used for treatment of osteoporosis (17) and in osteoporotic patients (18); it has been shown that bone resorption is reduced, while formation of bone is promoted (19). Strontium ranelate has been the most studied so far and has revealed positive outcomes in bone strength development $(18,19)$. While the ability of strontium citrate to induce bone healing has been well known, its efficacy in MOD has not been tested yet. This study aimed to assess the effects of strontium citrate during MDO in a rabbit model. The null hypothesis of this study is that using Strontium citrate during MDO will not reveal any differences on bone healing when compared to conventional MDO.

\section{MATERIALS AND METHODS}

This study was carried out on 14 adult healthy male white New Zealand rabbits. All rabbits were housed and cared for along with the Faculty of Agriculture on Laboratory animals. The study was accepted by the ethical committee of the Alexandria University Faculty of Dentistry.

\section{Materials}

\section{Experimental animals}

Rabbits weighed from 3 to $3.5 \mathrm{~kg}$ and were housed in individual cages, under climate-controlled conditions. They were allowed to acclimatize for 1 week before having surgery at the animal care facility. Randomly, the rabbits were divided into 2 separated equal groups; a study group (7 rabbits) which consumed [strontium citrate (675 mg / kg / day)] 7 days preoperative till 43 days postoperative and a control group ( 7 rabbits), which received no medication.

\section{Distraction device}

A custom-made stainless-steel distractor device $34 \mathrm{~mm}$ in total length and $3 \mathrm{~mm}$ in diameter manufactured and designed in cooperation with the Arab Engineers for Designs and Medical Equipment) (Fig. 1 a) appropriate for mandibles of rabbits, was designed to be positioned extra orally (subcutaneously). The overall distraction distance of $10 \mathrm{~mm}$ was set, and $0.5 \mathrm{~mm}$ of distraction was equal to every complete turn of the activation arm $1.9 \mathrm{~mm}$ self-tapping titanium screws fixed the distractor device to the mandible.

\section{Pharmaceutical Treatment}

The study drug strontium citrate (675 mg / kg / day) [(Life Extension Strontium Caps) National Nutrition (Orillia, Ontario, Canada)] was applied.

The rabbits were split to either consume the strontium citrate study drug (study group) or not to consume the strontium (control group).
The first group (study group) of rabbits consumed suspended oral strontium citrate administered through water bottles, which were carefully tested to ensure the whole medication dose $(675 \mathrm{mg} / \mathrm{kg}$ / day) was ingested daily. The overall time of the consumption of strontium citrate was 50 days, which divided into 7 days of preoperative adaptation, 5 days of latency stage, 10 days of distraction and 28 days of consolidation (20). To change the dosage as needed, the rabbits were weighed weekly.

The dose of strontium citrate was calculated and adjusted according to the rabbit weight and was based on previous studies of strontium in rabbits (20).

\section{Methods}

\section{Preoperative Care}

1- The animals were examined by a veterinarian to exclude any diseased animals.

2- The animals were weighed to estimate the amount of anesthetic solution.

3- The animals fasted the night before the surgical operation to prevent vomiting after anesthesia.

\section{Surgical Procedures}

The animals were injected intramuscularly with ketamine hydrochloride (Rotexmedica, Trit- tau, Germany) $35 \mathrm{mg} / \mathrm{kg}$ and xylazine (Xyla-ject; Adwia Pharmaceuticals SAE, Cairo, Egypt) $5 \mathrm{mg} / \mathrm{kg}$.

The surgical procedures were under aseptic conditions for all rabbits. The submandibular area, which is the operative site, was shaved and the area was scrubbed with Betadine (The Nile Co. pharmaceuticals) (Fig. 1 b). The incision line was marked, then the skin was incised by making extraoral submandibular incisions using Bard Parker scalpel blade no. 15. This incision was made on the inferior border of the mandible from the angle of the mandible to the anterior border of the mandible.

Dissection was carried out between the premolars and the incisors via the subcutaneous and the muscle layer, and the lateral side of the mandibular bone was visible. The mandible's mental neurovascular bundle was detected and preserved intact to protect the distracted bone's blood supply. The distractor was opened and adapted over the bone externally; the vertical osteotomy line was made on the mandibular cortex using a micro-reciprocating saw posterior to the mental foramen with copious irrigation using sterile saline (Fig. 1 c).

Four holes were made 2 on each side of corticotomy line, then the screws were tightened for fixation of the distractor using screws driver (Fig. 1 d).

The distractor device was then turned on for 1 to $2 \mathrm{~mm}$ in order to make sure that the osteotomy was completed, and the device was working. The incision was sutured under the activation arm of the distractor device by 4-0 vicryl (Fig.1 e), which was positioned in the center of the incision (20).

\section{Post-surgical Phase}

Postoperative antibiotics (intramuscular [i.m.] ceftriaxone 25mg/kg twice daily for 3 days) (SmithKline Beecham Egypt LLC. For: NOVARTIS PHARMA S.A.E.-Egypt. [SANDOZ]) and analgesics (i.m ketoprofen $2 \mathrm{mg} / \mathrm{kg}$ once per day and i.m. buprenorphine $0.04 \mathrm{mg} / \mathrm{kg}$ b.i.d., both for 3 days) (Sedico for pharmaceuticals. For: sanofi-aventis Egypt s.a.e.) were administered (20). 


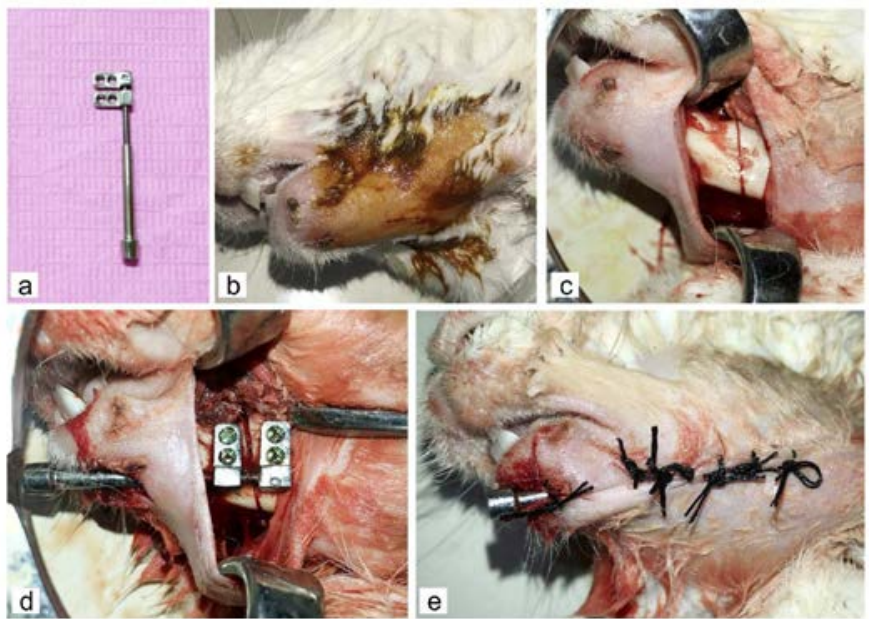

Figure (1): a. Custom made stainless-steel distractor.

b. Shaved submandibular area and painted with Betadine.

c. Corticotomy line determined and sawed with saline irrigation. d. Complete fixation of the distractor.

e. Skin stitched with4-0 vicryl suture.

The rabbits were moved to a clean cage were kept under observation until full recovery. On the first postsurgical day, the animals were given glucose water; then they were kept during the post- surgical period on a soft diet consisting of wet bread.

\section{Follow up period}

1- Animals were evaluated and tracked regularly until the completion of each group's follow-up period with respect to healing progress of the wound, ability to drink, eat and deviation of the mandible and any arising complications including signs of infection or edema.

2- Care of the wound was carried out daily with Betadine solution for 7 days and application of topical antibiotic spray.

3- Any changes in coat appearance, dietary patterns, or behavior were closely monitored.

The latency stage was 5 days; on the 6th post-surgical day, distraction using distractor activator was started at $1 \mathrm{~mm}$ for 10 days (total distraction of $10 \mathrm{~mm}$ ). After 4 weeks of consolidation, the rabbits were euthanized and the distracted mandibles were dissected and prepared for light microscopic evaluation and histomorphometric analysis.

Bone Analysis

Using the following studies, distracted mandibles were dissected and freshly obtained and defined.

\section{Histological preparation for light microscopy}

Specimens were labeled and fixed in $10 \%$ neutral buffered formalin. After fixation, specimens were decalcified, washed, dehydrated in ascending concentrations of ethanol, cleared with xylene, infiltrated and embedded in paraffin wax. Thin sections of $5 \mu \mathrm{m}$ thick were cut using a rotary microtome. Sections were stained with Haematoxylin \& Eosin stains (H\&E) for routine histological examination and Gomori's trichrome stain for evaluation of new bone formation by light microscope (21).

Histomorphometric analysis

Computer-assisted histomorphometry was performed to measure the percentage of new bone surface area per field formed in the two different groups (22). Sections at standardized depth were obtained and images were captured using the same magnification power (X100) with a digital camera coupled to an optical microscope.
The values were measured using an image processing program (Image J software, National Institutes of Health, USA).

\section{Statistical analysis of the data}

The data was fed to the computer and analyzed using version 20.0.0 of the IBM SPSS software package. (NY: IBM Corp, Armonk). In order to check the normality of distribution, the Kolmogorov-Smirnov test was used to classify the quantitative data using the range (minimum and maximum), mean, standard deviation, median and interquartile range (IQR). At the 5\% level, the significance of the results obtained was judged.

The used tests were (Student t-test)

To compare between two studied groups, for normally distributed quantitative variables.

\section{RESULTS}

\section{Histological results}

Examination of the histological sections stained by (H \& E) revealed new bone formation at the site of distraction. The newly formed trabecular bone showed relatively thin and scattered trabeculae (Fig. 2 a) surrounded by fibrous tissue (Fig. 3 a) with areas of immature bone (woven bone). Wide bone marrow spaces were also observed with areas of fatty tissue infiltration (Fig. 2 a and Fig. 3 a).

At the distraction site the study group revealed new trabecular bone formation, the newly formed bone showed interconnecting bony trabeculae lined by osteoblasts cells with normal sized, well vascularized bone marrow spaces (Fig. 2 b and Fig. 3 b). Evident fusion between old and new bone was observed (Fig. $\mathbf{3}$ b). Both the control and study groups showed wide osteocytes lacunae.

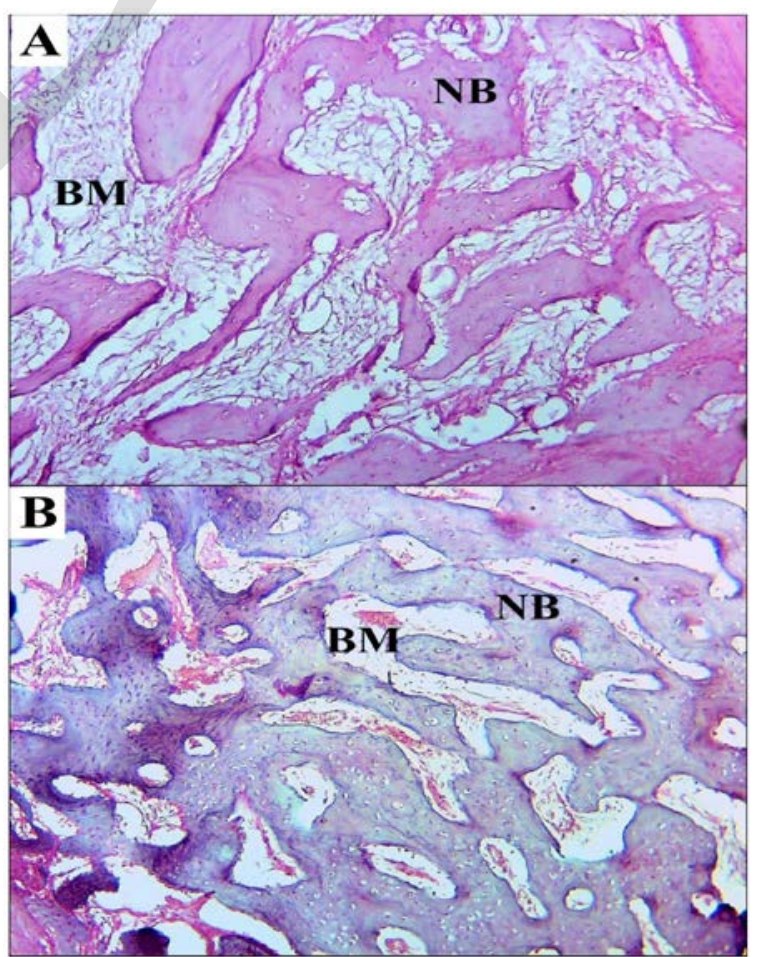

Figure (2): (A) Light micrograph (LM) Control group showing relatively thin and scattered spongy bone trabeculae with wide bone marrow. (B) LM Study group showing interconnected spongy bone trabeculae with well-vascularized marrow spaces. (H\&E X100) NB: new bone, BM: bone marrow. 


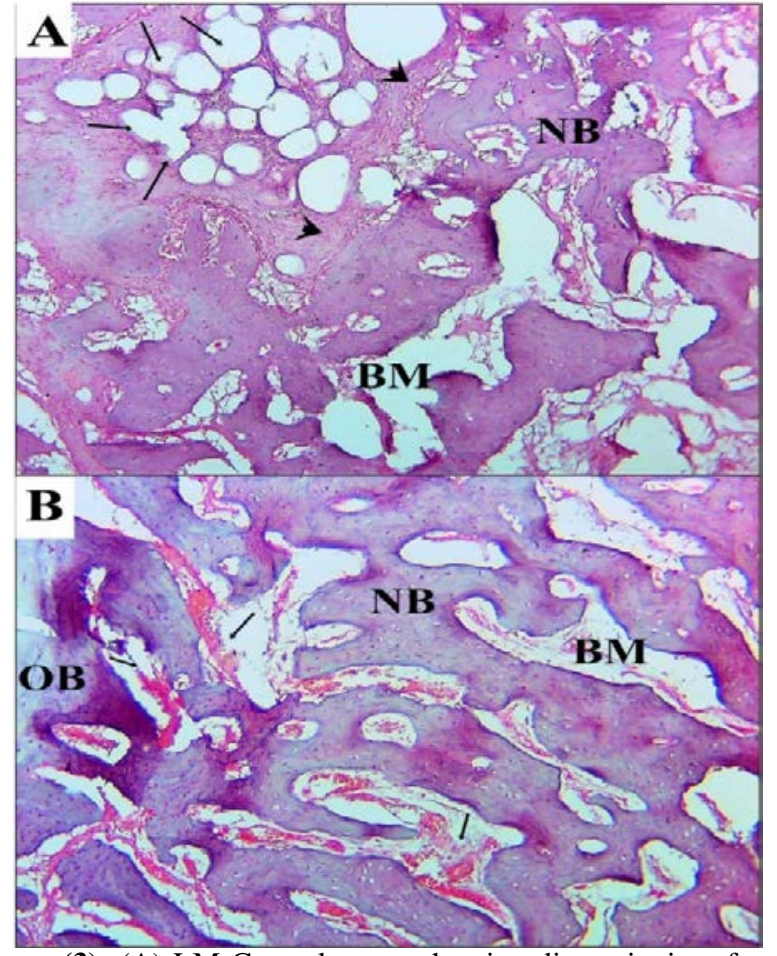

Figure (3): (A) LM Control group showing discontinuity of spongy bone trabeculae and wide bone marrow with fatty tissue infiltration (arrows) and areas of fibrous tissue (arrow heads). (B) LM Study group showing interconnected spongy bone trabeculae with well vascularized marrow spaces (arrows). (H\&E X100) NB: new bone, BM: bone marrow, OB: old bone.

Histological examination of the Gomori's Trichrome stained sections revealed similar results the H\&E stained sections, in both the control as well as the study group (Fig. 4 a, b and Fig. 5 a, b).

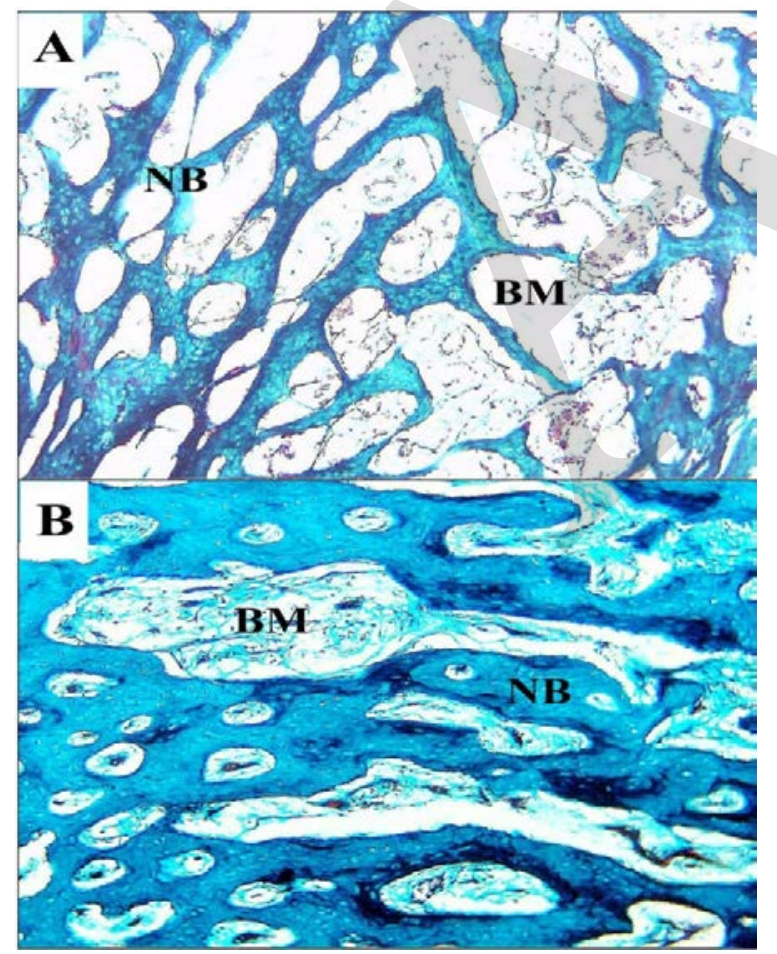

Figure (4): (A) LM Control group showing thin, widely separated spicules of immature bone. (B) LM Study group showing parallel spongy bone trabeculae with normal sized marrow spaces having the endosteal surface lined by osteoblasts. (Gomori’s TrichromeX100) NB: new bone, BM: bone marrow.

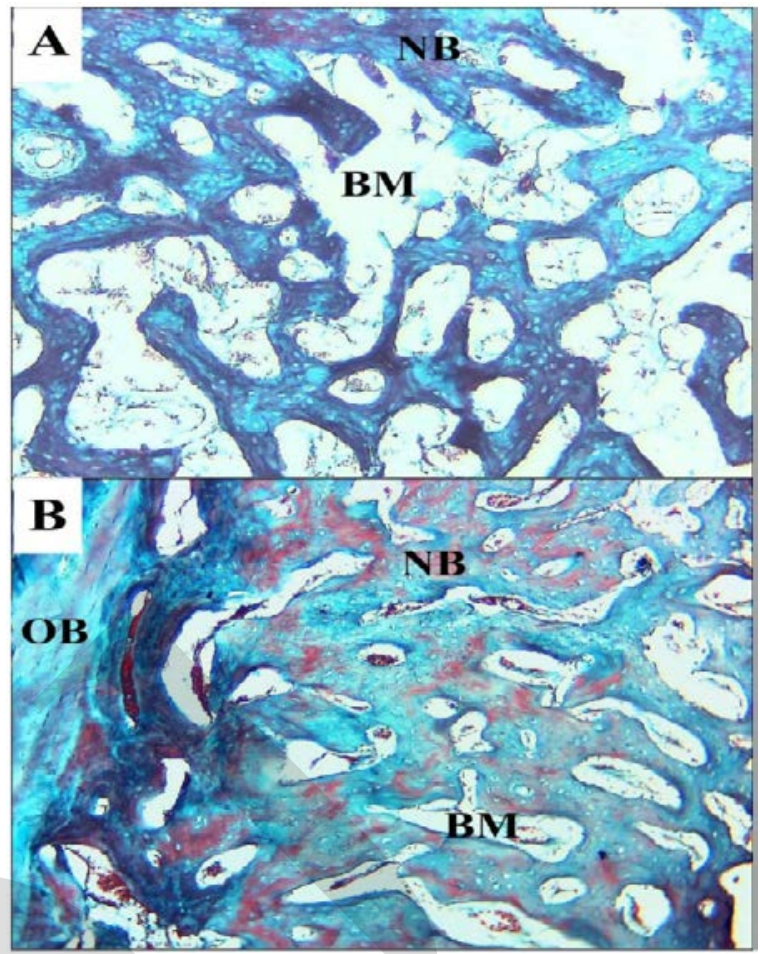

Figure (5): (A) LM Control group showing wide bone marrow spaces separating between thin bone trabeculae. (B) LM Study group showing new spongy bone trabeculae with normal sized marrow spaces; note the fusion between old bone and new bone. (Gomori's TrichromeX100) NB: new bone, BM: bone marrow, OB: old bone.

\section{Histomorphometric results}

The study group, which received strontium citrate, showed significant $(\mathrm{P}=0.001)$ _higher new bone formation than the control group. (Table 1, Fig. 6)

Table (1): Comparison between the two studied groups according to percentage of new bone per field

\begin{tabular}{|c|c|c|c|c||}
\hline $\begin{array}{c}\text { Percentage of } \\
\text { new bone per } \\
\text { field }\end{array}$ & $\begin{array}{c}\text { Control } \\
\mathbf{( n = 7 )}\end{array}$ & $\begin{array}{c}\text { Study } \\
(\mathbf{n}=7)\end{array}$ & $\mathbf{t}$ & $\mathbf{p}$ \\
\hline Min. - Max. & $45.69-63.36$ & $63.05-81.57$ & & \\
Mean \pm SD. & $55.49 \pm 6.95$ & $71.59 \pm 5.98$ & $4.647^{*}$ & $0.001^{*}$ \\
Median (IQR) & $58.42(50.3-60.2)$ & $71.70(68.4-74.0)$ & & \\
\hline
\end{tabular}

t: Student t-test

$\mathrm{p}: \mathrm{p}$ value for comparing between the studied groups

*: Statistically significant at $\mathrm{p} \leq 0.05$

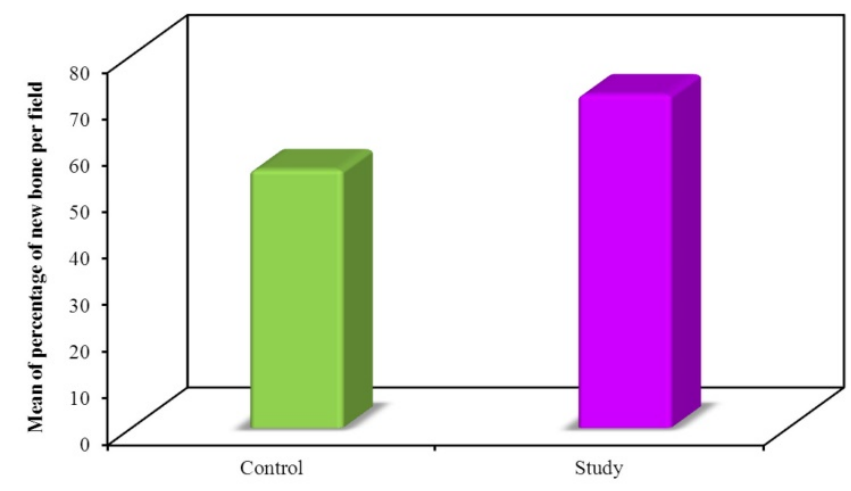

Figure (6): Comparison between the two studied groups according to percentage of new bone per field. 


\section{DISCUSSION}

Mandibular distraction animal models including monkey, minipig, rat, dog, and rabbit were developed and used over the previous 20 years in different studies. The use of rabbit model for the distraction of mandible came for its simple operating protocol with its cheap price, incidence shapes, moderate jaw size and its similarity to that of human bone on its peak bone mass (23).

Osteogenesis distraction has been an effective tool for the correction of craniofacial deformities. Mandibular distraction osteogenesis, however, requires prolonged period of treatment, which can lead to undesirable effects (9).

The bone healing efficacy of different adjuvant therapies in experimental MDO models has therefore been tested by research (12). Bisphosphonates (24), electrical stimulation (25), growth factors (26), hyperbaric oxygen (15), and lowintensity pulsed ultrasound stimulation (27) are some of the treatments reported to date.

While some of these adjuvant therapies with improved bone healing have shown positive results, limitations are present. For example, there are questions regarding the safety profile related to certain techniques (e.g., use of bisphosphonate osteomyelitis / osteonecrosis (28), and applied subjects remain (prices associated to the development of recombinant growth factors) (29), that stopped such treatments from occurring. In addition, certain adjuvant treatments need procedures that are invasive, for example, injecting growth factors into Callus, which repeated anesthesia in children.

On the other hand, strontium is considered as safe drug and may therefore be a more effective approach in the clinical setting to improve bone healing in MDO. Strontium is a chemical substance and alkaline earth metal with an atomic number 38. Strontium can be incorporated into the mineral phase of the bone remodeling due to the similarity to calcium ions (17), which used as a therapeutic medication in two types: strontium citrate, and strontium ranelate.

Strontium ranelate has been the most studied so far and has revealed positive outcomes in bone strength development (18, 19). The efficacy of the medication came from its ability to suppress osteoclast activity and promote osteoblast multiplication (30). Additionally, there were no recorded adverse effects or toxicity with use of strontium ranelate (17).

Furthermore, in the pediatric population, strontium ranelate is not an approved medication, that is necessary to note because MDO is frequently performed in infants with Robin sequence (9). Strontium is commonly considered to be the active substance, whereas the coupling substance or carrier molecule is the ranelate (17).

Definitely, strontium has an affinity for bone, as calcium (31). The current research, therefore, examined the efficacy of strontium citrate rather than strontium ranelate.

The present study was based on previous study including MDO Models of New Zealand White Rabbit (12, 32). In particular, the span of the latency period and the distraction frequency were selected to avoid premature consolidation of the callus tissue in the distraction region, which can prevent the effective separation of the bone segments $(23,33)$. Karp et al reported significant new bone formation at rates of $1 \mathrm{~mm} /$ day (34) which was consistent with the results of the current study however, Komuro et al., (35) reported that rate of $0,72 \mathrm{~mm} /$ day caused premature ossification of the regeneration zone.

More aggressive distraction rates were not used at the same time because they could result in osteotomized segments fibrosis or non-union leading to postoperative complications.

In the present study corticotomy was preferred to preserve the intermedullary blood supply, which was in agreement with Komuro et al., (35) who reported similar results in his study using cortictomy.

Our research has shown that in a rabbit model for MDO, strontium citrate effectively enhanced new bone formation (study group) in contrast to the results of the control group. The newly formed bone showed thick mature bone trabeculae. These results were obtained from microscopic and histological analysis. Our results are consistent with the results of Taylor et al. who revealed that new bone formation in the control group was sparse in comparison with the study group treated with strontium citrate in both H\&E and Masson's trichrome stained sections (20).

\section{CONCLUSION}

The result of the present study revealed that strontium citrate enhanced new bone formation and maturation in the study group in comparison to the control group. This finding suggests that strontium citrate supplementation could be beneficial during mandibular distraction osteogenesis. Further researches are recommended using different consolidation time and distraction distances.

\section{Conflict of interest}

No potential conflict of interest relevant to this article was reported.

\section{REFERENCES}

1. Ilizarov GA. Collected Scientific Works of the Kurgan Regional Scientific Medical Society. In: Ilizarov GA (ed). A new principle of osteosynthesis using crossing wires and rings. Kurgan: Union of Soviet Socialists Republic; 1954; pp.145-60.

2. Cao J, Zhang S, Gupta A, Du Z, Lei D, Wang L, et al. Sensory nerves affect bone regeneration in rabbit mandibular distraction osteogenesis. Int J Med Sci. 2019;16:831-7.

3. McCarthy JG, Schreiber J, Karp N, Thorne CH, Grayson $\mathrm{BH}$. Lengthening the human mandible by gradual distraction. Plast Reconstr Surg. 1992;89:1-8.

4. Molina F, Ortiz FM. Mandibular elongation and remodeling by distraction: a farewell to major osteotomies. Plast Reconstr Surg. 1995;96:825-40.

5. Alonso N. Alongamento osseo de mandibula no tratamento de assimetrias faciais. F Méd (Br). 1996;113:259-63.

6. Alonso N, Munhoz AM, Fogaça W, Ferreira MC. Midfacial advancement by bone distraction for treatment of craniofacial deformities. J Craniofac Surg. 1998;9:114-8.

7. McCarthy JG, Stelnicki EJ, Mehrara BJ, Longaker MT. Distraction osteogenesis of the craniofacial skeleton. Plast Reconstr Surg. 2001;107:1812-27.

8. Breugem CC, Evans KN, Poets CF, Suri S, Picard A, Filip $\mathrm{C}$, et al. Best practices for the diagnosis and evaluation of infants with Robin sequence: a clinical consensus report. JAMA Pediatr. 2016;170:894-902. 
9. Hong P. A clinical narrative review of mandibular distraction osteogenesis in neonates with Pierre Robin sequence. Int J Pediatr Otorhinolaryngol. 2011;75:985-91.

10. Hong P, Brake MK, Cavanagh JP, Bezuhly M, Magit AE. Feeding and mandibular distraction osteogenesis in children with Pierre Robin sequence: a case series of functional outcomes. Int J Pediatr Otorhinolaryngol. 2012;76:414-8.

11. Hong P, Kearns D. Airway characteristics of infants with Pierre Robin sequence who undergo mandibular distraction osteogenesis. Ear Nose Throat J. 2015;94:E25-9.

12. Hong P, Boyd D, Beyea SD, Bezuhly M. Enhancement of bone consolidation in mandibular distraction osteogenesis: a contemporary review of experimental studies involving adjuvant therapies. J Plast Reconstr Aesthet Surg. 2013;66:883-95.

13. Guo-ping W, Xiao-chuan H, Zhi-hui Y, Li G. Influence on the osteogenic activity of the human bone marrow mesenchymal stem cells transfected by liposome-mediated recombinant plasmid pIRES-hBMP2-hVEGF165 in vitro. Ann Plast Surg. 2010;65:80-4.

14. Zheng LW, Cheung LK. Effect of recombinant human bone morphogenetic protein-2 on mandibular distraction at different rates in a rabbit model. Tissue Eng. 2006;12:3181-8.

15. Salgado CJ, Raju A, Licata L, Patel M, Rojavin Y, Wasielewski S, et al. Effects of hyperbaric oxygen therapy on an accelerated rate of mandibular distraction osteogenesis. J Plast Reconstr Aesthet Surg. 2009;62:1568-72.

16. Appelman-Dijkstra NM, Papapoulos SE. Clinical advantages and disadvantages of anabolic bone therapies targeting the WNT pathway. Nat Rev Endocrinol. 2018;14:605-23.

17. Reginster JY, Lecart MP, Deroisy R, Lousberg C. Strontium ranelate: a new paradigm in the treatment of osteoporosis. Expert Opin Investig Drugs. 2004;13:857-64.

18. Meunier PJ, Roux C, Seeman E, Ortolani S, Badurski JE, Spector TD, et al. The effects of strontium ranelate on the risk of vertebral fracture in women with postmenopausal osteoporosis. N Engl J Med. 2004;350:459-68.

19. Delannoy P, Bazot D, Marie PJ. Long-term treatment with strontium ranelate increases vertebral bone mass without deleterious effect in mice. Metabolism. 2002;51:906-11.

20. Taylor BA, Bezuhly M, Brace M, Carter M, Hong P. Effect of strontium citrate on bone consolidation during mandibular distraction osteogenesis. Laryngoscope. 2017;127:E212-8.

21. Kumar G, Narayan B. Prevention of infection in the treatment of one thousand and twenty-five open fractures of long bones. Retrospective and prospective analyses. In: Classic Papers in Orthopaedics. London: Springer; 2014. pp. 527-30.

22. Eriksen EF, Axelrod DW, Melsen F. Bone histomorphometry. New York: Raven Press; 1994.
23. Djasim UM, Wolvius EB, van Neck JW, Weinans H, van der Wal KG. Recommendations for optimal distraction protocols for various animal models on the basis of a systematic review of the literature. Int $\mathrm{J}$ Oral Maxillofac Surg. 2007:36:877-83.

24. Tekin U, Tuz HH, Onder E, Ozkaynak O, Korkusuz P. Effects of alendronate on rate of distraction in rabbit mandibles. J Oral Maxillofac Surg. 2008;66:2042-9.

25. El-Hakim IE, Azim AM, El-Hassan MF, Maree SM. Preliminary investigation into the effects of electrical stimulation on mandibular distraction osteogenesis in goats. Int J Oral Maxillofac Surg. 2004;33:42-7.

26. Cho BC, Moon JH, Chung HY, Park JW, Kweon IC, Kim IS. The bone regenerative effect of growth hormone on consolidation in mandibular distraction osteogenesis of a dog model. J Craniofac Surg. 2003;14:417-25.

27. El-Bialy TH, Royston TJ, Magin RL, Evans CA, Zaki AelM, Frizzell LA. The effect of pulsed ultrasound on mandibular distraction. Ann Biomed Eng. 2002;30:125161.

28. Marx RE, Sawatari Y, Fortin M, Broumand V. Bisphosphonate-induced exposed bone (osteonecrosis/osteopetrosis) of the jaws: risk factors, recognition, prevention, and treatment. J Oral Maxillofac Surg. 2005;63:1567-75.

29. Jenkins MJ, Farid SS. Human pluripotent stem cell-derived products: advances towards robust, scalable and cost-effective manufacturing strategies. Biotechnol J. 2015;10:83-95.

30. Marie PJ, Felsenberg D, Brandi ML. How strontium ranelate, via opposite effects on bone resorption and formation, prevents osteoporosis. Osteoporos Int. 2011;22:1659-67.

31. Wohl GR, Chettle DR, Pejović-Milić A, Druchok C, Webber CE, Adachi JD, et al. Accumulation of bone strontium measured by in vivo XRF in rats supplemented with strontium citrate and strontium ranelate. Bone. 2013;52:63-9.

32. Zakhary K, Motakis D, Hamdy RH, Campisi P, Amar Y, Lessard ML. Effect of recombinant human bone morphogenetic protein 7 on bone density during distraction osteogenesis of the rabbit mandible. J Otolaryngol. 2005;34:407-14.

33. Stewart KJ, Lvoff GO, White SA, Bonar SF, Walsh WR, Smart RC, et al. Mandibular distraction osteogenesis: a comparison of distraction rates in the rabbit model. J Craniomaxillofac Surg. 1998;26:43-9.

34. Karp NS, Thorne CH, McCarthy JG, Sissons HA. Bone lengthening in the craniofacial skeleton. Ann Plast Surg. 1990;24:231-7.

35. Komuro Y, Takato T, Harii K, Yonemara Y. The histologic analysis of distraction osteogenesis of the mandible in rabbits. Plast Reconstr Surg. 1994;94:152-9. 